\title{
Design of an ultra-broadband power amplifier using distributed network synthesis
}

\author{
Nam-Tae Kim ${ }^{\mathrm{a}}$ \\ Department of Electronic Engineering, Inje University, Gimhae 621-749, Korea \\ a)elecknt@inje.ac.kr
}

Abstract: This paper presents a methodology for designing ultrabroadband power amplifiers (PAs) based on distributed network synthesis and offers impedance matching of a power device by parasitic absorption. For the synthesis of broadband matching circuits, transfer functions of distributed networks are provided using an equal-ripple approximation, and power device models are derived using negative-image modeling. An impedance-matching circuit that absorbs power device models can be synthesized by properly choosing the minimum insertion loss (MIL) and ripple parameters of the transfer function. As an example, distributed network synthesis is applied to the design of an ultra-broadband PA, and experiments are carried out.

Keywords: power amplifier, network synthesis, ultra-broadband Classification: Microwave and millimeter wave devices, circuits, and systems

\section{References}

[1] K. Niclas: IEEE MTT-S Int. Microwave Symp. Dig. (1984) 215.

[2] K. Niclas, W. Wilser, R. Gold and W. Hitchens: IEEE Trans. Microw. Theory Tech. 28 (1980) 285.

[3] Y. Ayasli, L. Reynolds, R. Mozzi and L. Hanes: IEEE Trans. Microw. Theory Tech. 32 (1984) 290.

[4] N. Sahan, M. Inal, S. Demir and C. Toker: IEEE Trans. Microw. Theory Tech. 56 (2008) 2032.

[5] D. Wu, F. Mkadem and S. Moumaiza: IEEE MTT-S Int. Microwave Symp. Dig. (2010) 1090.

[6] M. Mokari-Bolhassan and W. Ku: IEEE Trans. Microw. Theory Tech. 25 (1977) 837.

[7] R. Levy: IEEE Trans. Microw. Theory Tech. 20 (1972) 223.

[8] J. Helszajn: Synthesis of lumped element, distributed and planar filters (McGraw-Hill, New York, 1990) 286.

[9] N. Kim: Microwave Opt. Technol. Lett. 52 (2010) 2544.

[10] Agilent Technologies, Palo Alto, CA, U.S.A., 2005. 


\section{Introduction}

Design of ultra-broadband PAs is an important problem in applications including electronic warfare (EW), instrumentation, and software-defined radio (SDR). The challenge in designing broadband PAs is that power devices have impedances much lower than the system-level impedance, and it is not easy to match device impedances over a broad range of frequencies. Therefore, when combined with appropriate device-modeling techniques, designing ultra-broadband PAs becomes a matter of synthesizing impedance-matching networks to match power device models.

Several studies have been conducted to help in designing ultrabroadband PAs that can reach exacting performance levels. In $[1,2,3]$, lossy matched, feedback, and distributed-type amplifiers were investigated to solve such impedance-matching problems. Despite their great advantages, the methodologies provide poor output power and power-added efficiency. To overcome these issues, a broadband PA design that uses combined structures of lumped element networks and impedance transformers was proposed [4]. In that case, the methodology requires both impedance transformers and reactance-eliminating circuits because a lumped circuit removes the reactive part of a power device, and the transformer converts its real part to the system impedance. In [5], a broadband PA design was introduced using optimal-impedance analysis and simplified real-frequency techniques (SRFT). Although an SRFT approach can be used directly to synthesize a matching network from the impedance data of power devices, it requires rather complex procedures for optimizing the transfer function of matching circuits. The methodology uses lumped elements to produce impedance-matching networks, and when used in high-powered broadband applications, these lumped elements need to be converted to distributed elements. In ultra-broad-frequency cases, however, the conversion process can bring about errors in amplifier performance.

In this paper, we propose a methodology for designing ultra-broadband PAs that use distributed network synthesis and achieve impedance matching to a power device by parasitic absorption. For the sake of synthesizing broadband matching networks, equal-ripple transfer functions of distributed circuits are offered, and power device models are generated using negative-image techniques. A distributed network used for matching power device models can be designed by setting appropriate MIL and ripple values for the transfer function. As an application example, an ultrabroadband PA is designed using distributed network synthesis, and experiments are performed to verify the effectiveness of the proposed methodology.

\section{Distributed network synthesis}

Although narrowband circuits with well-defined structures can be constructed efficiently using alternative design procedures, it is not easy to find effective alternatives for synthesizing broadband networks. In the latter case, distributed network synthesis provides a useful methodology for designing ultra-broadband circuits.

The transfer function for a distributed network consisting of commen- 
surate transmission lines is given by [6]:

$$
\left|S_{21}(S)\right|^{2}=\frac{K\left(-S^{2}\right)^{m}\left(1-S^{2}\right)^{n}}{P_{n+r+m}\left(S^{2}\right)},
$$

which includes $n$ transmission line elements (TLEs), $r$ low-pass elements (LPEs), and $m$ high-pass elements (HPEs). In equation (1), $P_{n+r+m}\left(S^{2}\right)$ is a strictly Hurwitz polynomial of the order $n+r+m$ in $S^{2}$, and it must provide the desired type of approximation. $K$ is a gain parameter, and Rechards' variable $S$ is equal to $\Sigma+j \Omega$.

When the transfer function of a distributed network exhibits band-pass characteristics with equal ripple, then (1) becomes

$$
\left|S_{21}\right|^{2}=\frac{K}{1+\frac{\varepsilon^{2}}{2}\left[1+\cos \left(n \phi_{b}+r \xi_{1}+m \xi_{2}\right)\right]},
$$

where $\varepsilon$ is the ripple parameter of the function, and the network order $n+r+$ $m$ must be an even number. In equation (2), expressions for $\phi_{\mathrm{b}}$ and $\xi_{1}$ are derived from a quasi-low-pass mapping as follows [7]:

$$
\begin{gathered}
\cos \phi_{b}=\frac{\Omega^{2}\left(\Omega_{1}^{2}+\Omega_{2}^{2}+2\right)-\left(\Omega_{1}^{2}+\Omega_{2}^{2}+2 \Omega_{1}^{2} \Omega_{2}^{2}\right)}{\left(\Omega_{2}^{2}-\Omega_{1}^{2}\right)\left(1+\Omega^{2}\right)}, \\
\cos \xi_{1}=\frac{2 \Omega^{2}-\Omega_{1}^{2}-\Omega_{2}^{2}}{\Omega_{2}^{2}-\Omega_{1}^{2}},
\end{gathered}
$$

where $\Omega=\tan \theta=\tan \beta l, \Omega_{1}=\tan \theta_{1}$, and $\Omega_{2}=\tan \theta_{2}$. The angles $\theta_{1}$ and $\theta_{2}$ are electrical lengths of transmission lines at lower- and upper-band edge frequencies, respectively. To obtain an expression for $\xi_{2}$, we assume $\cos \xi_{2}=$ $\mathrm{A} / \Omega^{2}+\mathrm{B}$ for high-pass elements from (4). Solving for the constants $\mathrm{A}$ and B by constraining $\cos \xi_{2}$ to equal -1 at $\Omega_{1}$ and +1 at $\Omega_{2}$, we obtain

$$
\cos \xi_{2}=\frac{\Omega^{2}\left(\Omega_{1}^{2}+\Omega_{2}{ }^{2}\right)-2 \Omega_{1}{ }^{2} \Omega_{2}{ }^{2}}{\Omega^{2}\left(\Omega_{2}{ }^{2}-\Omega_{1}{ }^{2}\right)} .
$$

If we choose $m=0$ in (2), the band-pass network is reduced to a distributed quasi-low-pass circuit because the band-pass network is derived by adding HPEs to the low-pass circuit proposed by [7].

Additionally, when a distributed circuit exhibits equal ripple and highpass characteristics, (1) becomes [8]:

$$
\begin{gathered}
\left|S_{21}\right|^{2}=\frac{K}{1+\varepsilon^{2} \cos ^{2}\left(n \phi_{h}+m \xi_{h}\right)}, \\
\cos \phi_{h}=\frac{\sqrt{1+\Omega_{c}^{2}}}{\sqrt{1+\Omega^{2}}} \\
\cos \xi_{h}=\frac{\Omega_{c}}{\Omega}
\end{gathered}
$$

where $\Omega_{\mathrm{c}}=\tan \theta_{\mathrm{c}}$, and the angle $\theta_{\mathrm{c}}$ is an electrical length of transmission lines at the cut-off frequency of a high-pass network.

Reflection coefficients for (2) and (6) are determined by the unitary 
condition for a lossless network, and the corresponding impedance function is given by

$$
z(S)=\frac{1+S_{11}(S)}{1-S_{11}(S)}
$$

where $S_{11}(S)$ is the input reflection coefficient, and $z(S)$ is the normalized input impedance for a distributed circuit. By choosing the proper MIL and ripple parameters for the transfer function of a given electrical length, we can synthesize the broadband impedance-matching network for any desired performance level.

\section{Ultra-broadband PA}

A design example is presented to illustrate the distributed network synthesis method and its application to ultra-broadband PA design. For SDR applications, a PA is required to have a small-signal gain greater than $18 \mathrm{~dB}$ and an output power greater than $39.55 \mathrm{dBm}$ at $1-\mathrm{dB}$ gain compression over a frequency range of 0.5 to $2.5 \mathrm{GHz}$. It should also exhibit two-tone third-order intermodulation (IM3) of less than $-36 \mathrm{dBc}$, for a total output power of $33 \mathrm{dBm}$ with $10 \mathrm{MHz}$ tone spacing. The power device used in the amplifier is a GaN HEMT (CGH40010F) from Cree.

\subsection{Power device models}

Most power device manufacturers routinely provide designers with nonlinear models of power devices and device impedances associated with specific amplifier performance. Therefore, if amplifier performance targets are given, we can derive the desired power device models by using negativeimage modeling to find the impedances that offer the required circuit performance [9]. Fig. 1 (a) shows the CGH40010F device with a shunt resistor for amplifier stability, which operates at a bias of $\mathrm{V}_{\mathrm{ds}}=28$ Volts and $\mathrm{I}_{\mathrm{ds}}=800 \mathrm{~mA}$. Fig. 1 (b) shows the equivalent input and output device models for the circuit of Fig. 1 (a) that were derived to provide the abovementioned amplifier performance by using the negative-image approach. The two-element port descriptions consisting of a resistor and a reactive element provide a convenient starting point for synthesizing broadband matching networks that can absorb the device model.

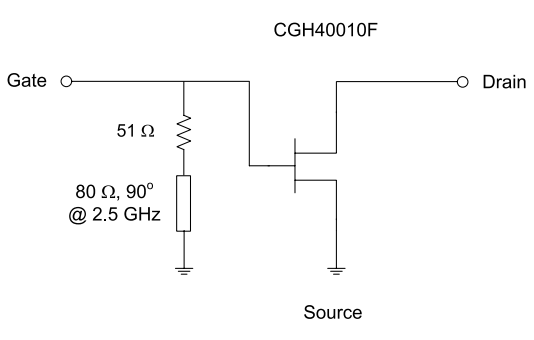

(a)

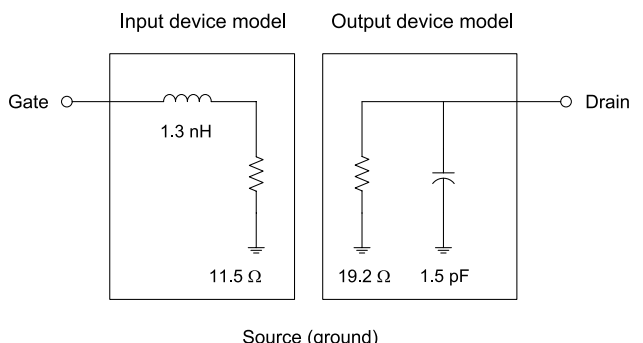

(b)

Fig. 1. (a) Power transistor with a stabilizing resistor;

(b) equivalent input and output device models of Fig. 1 (a). 


\subsection{Matching network synthesis}

In this section, distributed impedance-matching networks are synthesized to match the input and output device models using parasitic absorption. The matching circuits are designed to have greater bandwidths than the operating frequency range, which is done to accommodate bandwidth variations caused by implementation tolerances of the networks.

Because the output device model of the power device contains a shunt capacitor, an output-matching network can be constructed using band-pass or quasi-low-pass structures. Because a band-pass circuit provides shuntshorted stubs for DC bias injection, we synthesize an output network using a band-pass structure. To design an output-matching circuit, we choose $n=$ $2, r=3, m=1$, and $\theta_{2}=67^{\circ}$ in $(2)$ over a 0.5 to $2.6 \mathrm{GHz}$ range and then adjust MIL and ripple parameters to create a network for absorbing the output device model. Setting the MIL to $0.17 \mathrm{~dB}$ and the ripple to $0.42 \mathrm{~dB}$, $\left|S_{21}(S)\right|^{2}$ is calculated from (2), and $\left|S_{11}(S)\right|^{2}$ is obtained by the unitary condition. If we synthesize the output circuit as a minimum-phase network, $S_{11}(S)$ is determined from the poles and zeros of $\left|S_{11}(S)\right|^{2}$ in the left half of the S-plane. The impedance function for the output matching circuit is calculated from (9). By extracting the elements of a network topology that includes the output device model, we can synthesize a distributed network for transforming the system impedance of $50 \Omega$ to the load resistance of 19.2 $\Omega$. Fig. 2 (a) shows a synthesized output-matching circuit, where the open stub adjacent to the resistor of $19.2 \Omega$ is divided into a shunt capacitor and the portion of the open stub left after removal of the capacitance made with the purpose of absorbing the output device model shown in Fig. 1 (b).

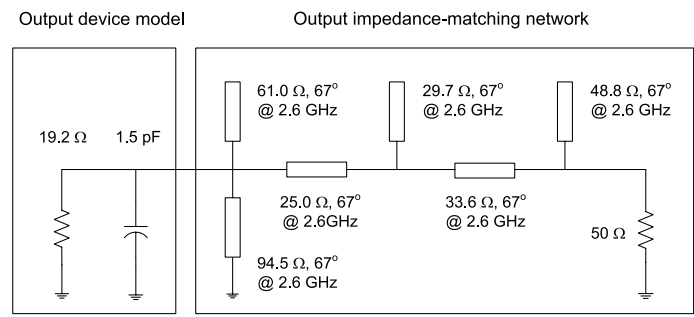

(a)

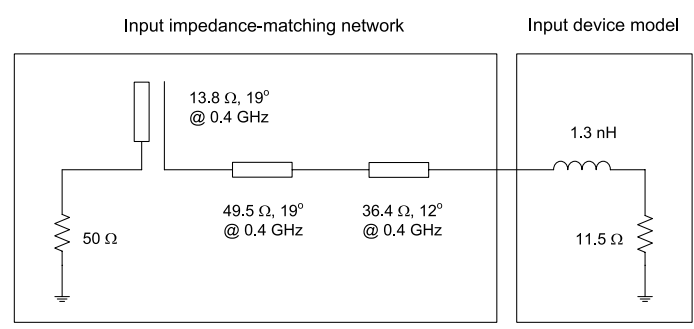

(b)

Fig. 2. Synthesized networks: (a) output-matching network $\left(n=2, r=3, m=1\right.$, and $\left.\theta_{2}=67^{\circ}\right)$; (b) inputmatching network $\left(n=2, m=1\right.$, and $\left.\theta_{\mathrm{c}}=19^{\circ}\right)$.

An input-matching network can also be designed by the same procedure. To suppress low-frequency gain of the power device, we synthesize the input network using a high-pass structure. For the inputmatching circuit, we choose $n=2, m=1$, and $\theta_{c}=19^{\circ}$ at a cut-off frequency 
of $0.4 \mathrm{GHz}$ in (6). If we set MIL to $0.768 \mathrm{~dB}$ and the ripple to $0.61 \mathrm{~dB}$, $\left|S_{21}(S)\right|^{2}$ is determined from (6), and $\left|S_{11}(S)\right|^{2}$ is obtained from the unitary condition. If the input network is also synthesized as a minimum-phase network, $S_{11}(S)$ is then calculated from the poles and zeros of $\left|S_{11}(S)\right|^{2}$ in the left half of the S-plane, and the impedance function is obtained from (9). By extracting network elements in a topology meant to absorb the input device model, we can synthesize a distributed circuit that transforms resistance of $50 \Omega$ to resistance of $11.5 \Omega$. Fig. 2 (b) shows a synthesized input-matching network, where the TLE adjacent to the resistor of $11.5 \Omega$ is split into a series inductor and the portion of the TLE remaining after removal of the inductance made with the purpose of absorbing the input device model shown in Fig. 1 (b). Because the distributed network synthesis approach provides considerable flexibility in selecting a circuit topology and adjusting parasitic element values, it offers an effective methodology for designing broadband impedance-matching networks.

\subsection{PA performance}

A PA built using the combined techniques of section 3.1 and 3.2 is shown in Fig. 3. Here, the stubs that provide DC bias injection are RF shorted by shunt capacitors, and the output-matching network is designed to include a DC blocking capacitor. The series open stub in the input-matching circuit is replaced by a dual-function impedance-matching and DC-blocking capacitor, using a conversion between lumped and distributed elements [7]. The amplifier is optimized for the desired performance based on the synthesized element values in Fig. 2.

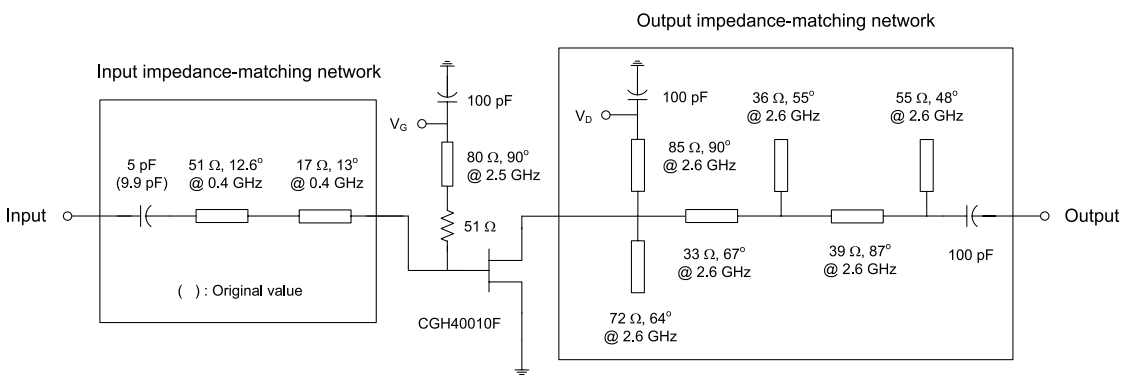

Fig. 3. Optimized ultra-broadband PA.

The PA circuit shown in Fig. 3 was realized on a TLX-9 copper-clad substrate from Taconic, with substrate thickness of $0.508 \mathrm{~mm}$, conductor thickness of $0.018 \mathrm{~mm}$, dielectric constant of 2.5 , and loss tangent of 0.0019 at $10 \mathrm{GHz}$. Fig. 4 shows the fabricated amplifier.

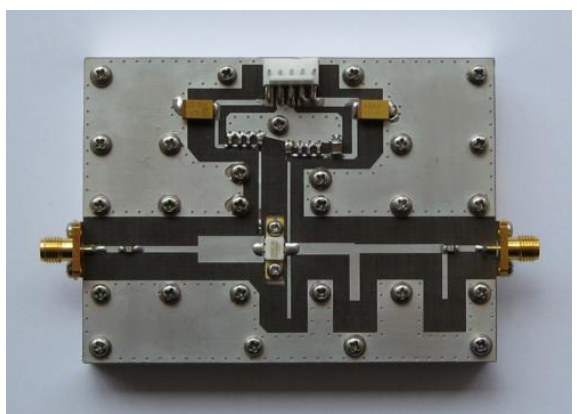

Fig. 4. Fabricated ultra-broadband PA. 
To consider the effect of micro-strip discontinuities, dielectric loss, and conductor loss, performance simulations were carried out using small-signal S-parameter and harmonic-balance analysis routines in a commercial microwave computer-aided design (CAD) software package (Agilent Technologies Advanced Design System 2005A [10]). Fig. 5 presents the simulated performance of the PA. In the simulations, the small-signal gain of the amplifier is $19.5 \pm 0.6 \mathrm{~dB}$, and the output power is $41.14 \pm 0.87 \mathrm{dBm}$ at 1 -dB gain compression over a 0.5 to $2.5 \mathrm{GHz}$ frequency range. Its IM3 is less than $-44.9 \mathrm{dBc}$, for a total output power of $33 \mathrm{dBm}$ with $10 \mathrm{MHz}$ tone spacing across the operating frequency range.

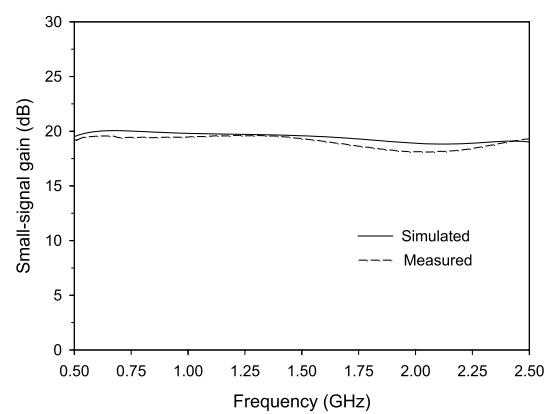

(a)

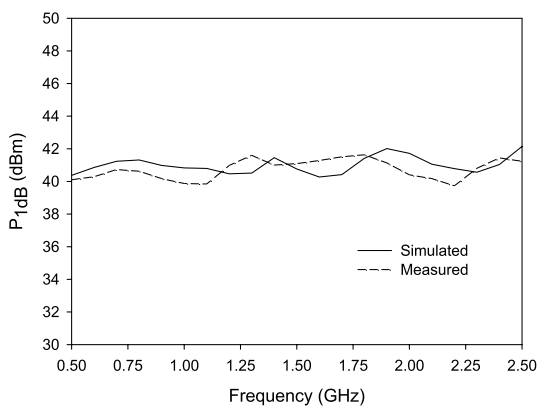

(b)

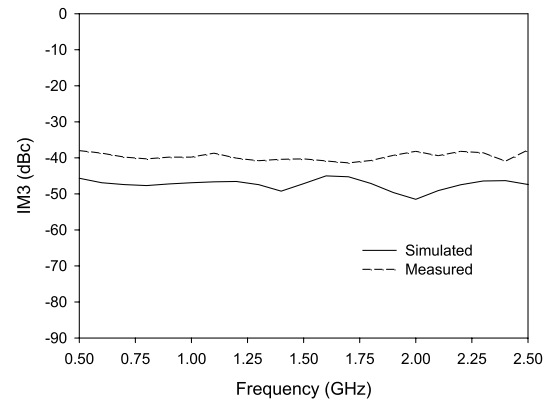

(c)

Fig. 5. Simulated and measured performance of the designed PA: (a) small-signal gain; (b) output power at 1-dB gain compression; (c) two-tone IM3 for a total output power of $33 \mathrm{dBm}$ and $10 \mathrm{MHz}$ tone spacing.

The measured performance of the fabricated PA is also shown in Fig. 5. The small-signal gain is $18.9 \pm 0.7 \mathrm{~dB}$, and the output power is $40.7 \pm$ $0.95 \mathrm{dBm}$ at 1 -dB gain compression over the same frequency range. Its twotone IM3 for the above-mentioned conditions is less than $-37.7 \mathrm{dBc}$ across the operating frequency range. These experimental results satisfy the required design goals.

\section{Conclusion}

A methodology that uses distributed network synthesis and offers impedance matching of a power device by parasitic absorption has been described for designing ultra-broadband PAs. The equal-ripple transfer function of a distributed circuit was provided to synthesize broadband 
matching networks, and power device models for parasitic absorption were derived using negative-image techniques.

As an example, distributed network synthesis was applied to the design of an ultra-broadband PA, and experiments were carried out. Test results confirm that distributed network synthesis is an effective methodology for designing ultra-broadband PAs.

\section{Acknowledgments}

This work was supported by the 2013 Inje University research grant. 